\title{
The time course of morphological processing during spoken word recognition in Chinese
}

\author{
Wei Shen ${ }^{1,2} \cdot$ Qingqing $\mathrm{Qu}^{1,2} \cdot$ Aiping $\mathrm{Ni}^{1,2} \cdot$ Junyi Zhou ${ }^{1,2} \cdot$ Xingshan $\mathrm{Li}^{1,2}$
}

Published online: 30 March 2017

(C) Psychonomic Society, Inc. 2017

\begin{abstract}
We investigated the time course of morphological processing during spoken word recognition using the printedword paradigm. Chinese participants were asked to listen to a spoken disyllabic compound word while simultaneously viewing a printed-word display. Each visual display consisted of three printed words: a semantic associate of the first constituent of the compound word (morphemic competitor), a semantic associate of the whole compound word (whole-word competitor), and an unrelated word (distractor). Participants were directed to detect whether the spoken target word was on the visual display. Results indicated that both the morphemic and whole-word competitors attracted more fixations than the distractor. More importantly, the morphemic competitor began to diverge from the distractor immediately at the acoustic offset of the first constituent, which was earlier than the wholeword competitor. These results suggest that lexical access to the auditory word is incremental and morphological processing (i.e., semantic access to the first constituent) that occurs at an early processing stage before access to the representation of the whole word in Chinese.
\end{abstract}

Keywords Morphology · Spoken word recognition . Chinese $\cdot$ Printed-word paradigm

Many words are composed of more than one morpheme in alphabetic and logographic writing systems. These words are

Xingshan Li

lixs@psych.ac.cn

1 Key Laboratory of Behavioral Science, Institute of Psychology, Chinese Academy of Sciences, 16 Lincui Road, Beijing, China

2 Department of Psychology, University of Chinese Academy of Sciences, Beijing, China usually called morphologically complex words. Individual morphemes are accessed during the processing of morphologically complex words (Hyönä \& Pollatsek, 1998; Pollatsek, Hyönä, \& Bertram, 2000). However, the question of whether individual morphemes are accessed before, after, or simultaneously with the activation of whole-word representation remains unanswered. This study aimed to investigate the time course of morphological processing during spoken word recognition in Chinese. Specifically, we studied whether semantic access of the first constituent of a compound word occurs before, after, or simultaneously with the access of the whole word during spoken word recognition in Chinese.

Compared with alphabetical languages, Chinese is characterized by several unique properties that may affect the processing of morphologically complex words. For instance, inflectional and derivational morphologically complex words are scarce in Chinese, and more than $70 \%$ of Chinese words are disyllabic compound words (Zhou \& Marslen-Wilson, 2000). The morpheme boundary in alphabetical languages does not always coincide with the syllable boundary, especially when the second morpheme begins with a vowel. In Chinese, one morpheme is mapped directly onto one syllable. Therefore, a clear morpheme/syllable boundary can be observed in morphologically complex Chinese words (Zhou \& Marslen-Wilson, 2000). For instance, a word such as 草率 (cao3shuai4, careless) is composed of two morphemes (syllables), and the morpheme/ syllable boundary is located between the two morphemes/syllables草(cao3, grass) and 率(shuai4, lead). Thus, the morpheme/syllable boundary is more salient as compared with alphabetic languages, making Chinese an ideal language to use in exploring the morphological processing. This saliency feature may facilitate morphological decomposition during word recognition in Chinese (Tsang \& Chen, 2014).

The models of morphologically complex word processing can be categorized into three models based on assumptions 
regarding the time course of morphological processing. A sublexical model assumes that morphologically complex words are decomposed obligatorily; thus, morphemes are activated before whole-word representation is activated (Taft \& Forster, 1975). A supralexical model postulates that morphemes are activated only after whole-word representation is activated (Giraudo \& Grainger, 2000). Dual route models assume that two parallel routes, namely, a decompositional route and a direct whole-word access route exist, and that these two routes compete with each other during the processing of morphologically complex words (Caramazza, Laudanna, \& Romani, 1988; Pollatsek et al., 2000). Factors such as word familiarity, word frequency, and semantic transparency likely influence which route wins.

For visual word recognition, some studies have shown that a morphologically complex word is accessed via the decompositional route in the early processing stage, which supports the sublexical model. Using the masked priming paradigm, Rastle, Davis, and New (2004) found that a briefly (42 $\mathrm{ms}$ ) presented transparent derivation prime (e.g., cleaner) facilitated the recognition of a stem target (e.g., CLEAN) equivalent to that of a pseudomorphologically complex word (e.g., corner) priming its stem (e.g., CORN), but they did not observe similar facilitation in the orthographic control group (e.g., brothelBROTH). They argued that morphological decomposition occurs at a very early stage, and any words with superficial morphological structure are possibly decomposed into individual constituents. Giraudo and Grainger (2000) found evidence against the sublexical model. Using an identical task as that in Rastle et al. (2004), they found that derived French target words, such as lait.ier (means "dairy cow"; morpheme boundaries are marked by dots), are primed with true morphological primes, such as lait.age ("dairy products"), but not with pseudoroot primes, such as laitue ("lettuce"; where/lati/is not a true stem) as compared with unrelated primes. The authors indicated that this finding supported a supralexical account of morphological representation. Relevant evidence from eye-movement studies have shown that the decompositional route is not the only way that morphologically complex words are accessed. Those words can also be accessed through the whole-access route, which occurs parallel to the decompositional route based on the finding that the whole-word frequency effect occurs at least as rapidly as the frequency effect of the second morpheme (Pollatsek et al., 2000).

In contrast to visually presented words, spoken signals unfold gradually over time. As such, while spoken information is accessed incrementally and continuously, whole-word information cannot be perceived until the second constituent becomes available. Hence, morphological decomposition may occur before the lexical access of a whole word in spoken word recognition.

Studies on the time course of morphological processing in spoken word recognition have not reached similar conclusions. Some studies have shown that morphological decomposition occurs after lexical access. Isel, Gunter, and Friederici (2003) examined the role of the first constituent during the spoken word processing of a German compound word. They instructed participants to complete a lexical decision task on a visual target word, which was either a semantic associate (e.g., Besuch-"visit") of the first constituent of spoken words (e.g., Gasthaus-"guesthouse") or a nonsemantic associate. They also manipulated visual words to be presented at either the offset of whole spoken words (Experiment 1) or the offset of the first constituent of the spoken words (Experiment 2). Experiment 1 demonstrated a significant priming effect, that is, the semantic associate of the first constituent yielded shorter reaction time than the unrelated one. Conversely, Experiment 2 revealed the absence of this priming effect on the first constituent. Hence, they concluded that semantic access to the first constituent occurs after the activation of the whole-word representation but not during online processing.

However, some studies have also demonstrated that morphological decomposition occurs before a whole word is accessed. Holle, Gunter, and Koester (2010) argued that compound words with short word length may be responsible for the absence of the priming effect of the first constituent in Isel et al. (2003) because longer words are more likely to yield larger priming effects. Therefore, they investigated the processing of long German compound words by using a semantic relatedness judgment task combined with ERP recording. They manipulated the lexical status of each constituent of compound words to be either "word" or "nonword" and established four conditions: word + word (e.g., ant|hill), word + nonword (e.g., machine|bönf), nonword + word (e.g., patose/chimney), and nonword + nonword (e.g., kronube(josche). They found a larger N400 amplitude at the offset of the first constituent for the nonword initial constituent than that for the word initial constituent. This finding indicates that semantic access to a nonword constituent is more difficult than semantic access to a word constituent. Hence, this finding can be considered as direct evidence for the occurrence of the early morphological decomposition before whole-word representation is accessed.

Zhou and Marslen-Wilson (1994) investigated morphological processing in Chinese spoken word recognition. They manipulated whole-word frequency, morpheme frequency, and syllable frequency ${ }^{1}$ of both constituents of a disyllable compound word by using an auditory lexical decision task. They found significant whole-word frequency effect (highwhole-word-frequency words produce shorter reaction times than low-whole-word-frequency words) but did not observe

\footnotetext{
${ }^{1}$ Syllable frequency refers to the occurrence of a syllable that corresponds to a given morpheme. This parameter is described by Zhou and Marslen-Wilson (1994) in detail.
} 
any morpheme or syllable effects. On the basis of these findings, Zhou et al. (1994) argued that whole-word representation plays a major role in the access of compound words.

Our study aimed to investigate the time course of the morphological processing in spoken Chinese word recognition by using a printed-word version of a visual-world paradigm. This paradigm can provide close time-locked measures and reflect online cognitive processing with spoken words unfolding over time (Huettig \& McQueen, 2007). Shen, Qu, and Li (2016) showed that a printed-word paradigm is applicable for use in investigating semantic information processing during Chinese spoken word recognition. They instructed participants to listen to a spoken target word embedded in a neutral sentence and view a printed word visual display, including a semantic competitor (i.e., the word was semantically related to the spoken target word) and two unrelated distractors. They observed that the fixation probability on semantic competitors is significantly higher than that on distractors. Hence, semantic information in spoken word recognition can mediate a visual attention shift to printed Chinese visual words.

In this study, participants were asked to listen to a spoken disyllable target word while looking at a visual display composed of three printed words. The printed words were manipulated to be a semantic associate of the first constituent (morphemic competitor), a semantic associate of the target word (whole-word competitor), or an unrelated word (distractor). The participants were instructed to determine whether the target was on the visual display. Their eye movements were recorded simultaneously. If morphological processing is an early process before lexical access to the whole word according to the sublexical model, then a morphemic competitor effect (the morphemic competitor attracted more fixations than the distractor did) should emerge earlier than a wholeword competitor effect (whole-word competitor attracted more fixations than the distractor). A late morphemic competitor effect or after whole-word competitor effect was expected, thereby supporting the supralexical model.

\section{Method}

\section{Participants}

Thirty-seven undergraduate students from universities near the Institute of Psychology, Chinese Academy of Sciences, were paid to participate in the experiment. These participants are all fluent Mandarin Chinese speakers ${ }^{2}$ and had normal or

\footnotetext{
${ }^{2}$ Some participants grew up in families that speak in a Chinese dialect. However, all of them are educated in Mandarin and are exposed to Mandarin (e.g., TV and radio) from a young age, and thus have no difficulty in listening and speaking Mandarin.
}

corrected-to-normal vision. They were unaware of the purpose of the experiment.

\section{Materials and design}

In Chinese, a large number of homophones are used so that one syllable may correspond to more than one morpheme. Hence, to avoid ambiguity, we very carefully selected the first syllables so that each syllable corresponded to one dominant morpheme. Twenty participants were instructed to write down the first morpheme that came into their minds for each visually presented syllable plus a tone (e.g., ma3). A syllable was selected if more than $80 \%$ of the participants reached an agreement on the same morpheme. Finally, 36 syllables were selected (mean agreement $=.94)$. Each morpheme was combined with another morpheme to constitute a disyllable compound word. To distinguish between morphological and whole-word processing, we manipulated the first morpheme (e.g., “草”,/cao3/, grass) to be semantically unrelated to the meaning of the whole word (e.g., “草率”,/cao3shuai4/, careless). Each display consisted of three printed words: (a) a morphemic competitor, which was a semantic associate of the first constituent of the spoken target word (e.g., “树木”,/ shu4mu4/, tree); (b) a whole-word competitor, which was a semantic associate of the spoken target word (e.g., “谨慎', jin3shen4/, cautiousness); and (c) a distractor, which was neither semantically related to the first constituent nor to the spoken target word (e.g., “祖父”, zu3fu4, grandpa; see Fig. 1). The printed words under these conditions were matched on word frequency and number of strokes. The printed words did not also share phonological information with spoken words. Most of the printed words ${ }^{3}$ did not share orthographic information with spoken words. No target words were included in the critical trials, but target words were included in the same number of filler trials.

A semantic relatedness rating was performed to ensure that the preference for eye fixation on visual display is unaffected by the semantic relatedness between spoken and printed words. Results have shown that semantic relatedness score of first morpheme versus morphemic competitor did not differ from the target word versus whole-word competitor $(t<1)$.

\section{Apparatus}

Eye movements were recorded using an EyeLink1000 tracker that takes samples at $1000 \mathrm{~Hz}$. Experimental materials were presented on a 21-inch CRT monitor. The participants were seated $58 \mathrm{~cm}$ from the video monitor. All visual printed words

\footnotetext{
${ }^{3}$ Four printed words were orthographically similar with the spoken target words (i.e., sharing a radical). The results did not change after these four items were excluded from analyses. This finding was in line with the Shen et al. (2016), which found that the semantic competitor effect was not modulated by orthographic similarity in Chinese.
} 


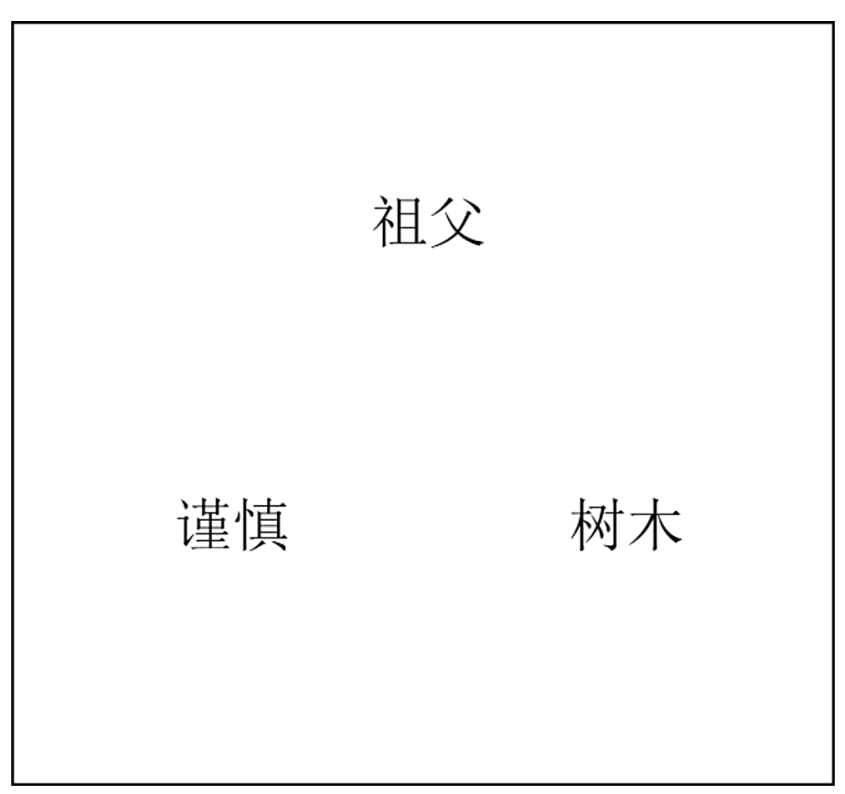

Fig. 1 Example of a printed-word display. For a spoken target word “草 率" (/cao3shuai4/, careless), the printed-word display consisted of a morphemic competitor word “树木” (/shu4mu4/, tree), a whole-word competitor word“谨慎” (/jin3shen4/, cautiousness) and a distractor“祖 父” (/zu3fu4/, grandpa) in three different positions of the display

were presented in a 30 Song font, with each character subtending a visual angle of approximately $1.4^{\circ}$ and appearing approximately $5^{\circ}$ from the center of the screen at this viewing distance. Eye movement data were collected only from the right eye.

\section{Procedure}

All spoken target words were recorded in a natural manner by a female native Chinese speaker. The spoken target words were presented to the participants through a headphone.

Nine-point calibration was conducted at the beginning of the experiment, and whenever the calibration error was more than $1^{\circ}$. A drift check procedure was performed at the beginning of each trial. Afterward, a blank screen was presented for $500 \mathrm{~ms}$, and then the visual display was previewed for 200 $\mathrm{ms}^{4}$ before the onset of the spoken target words. The participants were instructed to decide whether the printed word referred to by the spoken target word was on the display by pressing buttons. The visual display remained on the screen until a response was made. In this study, we only analyzed data from trials when no target word was presented during the visual display. Hence, "NO" and "YES" responses were required for critical and filler trials, respectively. The three printed words were arranged in a V-shape or an inverted $\mathrm{V}$-shape. The positions of the three printed words were

\footnotetext{
${ }^{4}$ Shen et al. (2016) showed that preview time of the visual display did not influence semantic information processing, and hence only a short preview condition was included in this study.
}

randomized across trials. The participants' eye movements were recorded simultaneously.

Initially, 20 practice trials were presented to familiarize the participants with the experimental procedure. They subsequently participated in a total of 72 experimental trials with intermixed critical and filler trials. The entire experiment lasted about 15 minutes.

\section{Results}

Incorrect trials $(0.3 \%)$ with wrong responses were excluded from the analyses. Fixation was defined as fixating on a word if it was within a square of $5^{\circ} \times 5^{\circ}$ at the center of the printed word. Figure 2 shows the fixation proportions of the morphemic competitor, whole-word competitor, and distractor calculated every $100 \mathrm{~ms}$ from $200 \mathrm{~ms}$ before the onset of the spoken target words and 1,500 ms after the onset of the target words. Fixations on the morphemic competitors indicated an early divergence from the distractor from almost $500 \mathrm{~ms}$ after the onset of the spoken words. The curve of the fixation proportion on whole-word competitor also exhibited a moderate increase relative to distractor from almost $600 \mathrm{~ms}$ after the onset of the spoken words.

Logit mixed models were employed to analyze the fixation proportion data (Jaeger, 2008). A dependent variable was defined as whether a fixation was made to a printed word within a specific time window. In particular, we were interested in the time course of the morphological processing. As such, we analyzed the time course for each time window of $100 \mathrm{~ms}$. Considering that about $150-200 \mathrm{~ms}$ is necessary to initiate eye movement (Rayner, 1998) and that the average duration of the spoken target word is about $750 \mathrm{~ms}$, we analyzed eight $100 \mathrm{~ms}$ time windows from $200 \mathrm{~ms}$ after the onset of spoken target word and $200 \mathrm{~ms}$ after offset of spoken target onset (i.e., $200 \mathrm{~ms}$ to $1,000 \mathrm{~ms}$ ). Bonferroni correction was performed to avoid the problem of multiple comparisons. Hence, only corrected $p$ values are reported in Table 1 .

For each analysis in each time window, random intercepts for participants and items and by-participants random slopes for competitor type were included as random effects (Barr, Levy, Scheepers, \& Tily, 2013). Competitor type was entered as fixed effects. In the models, the distractor was assigned as baseline as compared with the morphemic and whole-word competitors. The model was fitted by using the glmer function from lme4 package (Version 1.1-7; Bates, Maechler, Bolker, \& Walker, 2014) in an R environment (Version 3.3.2; R Core Team, 2016). Regression coefficients $b$, standard errors $S E, Z$ values, and $p$-values were reported. The model was fitted by creating a base model consisting of intercepts for participants and items as random effects. The model was enriched by adding competitor type and by-participants random slopes for competitor type. 


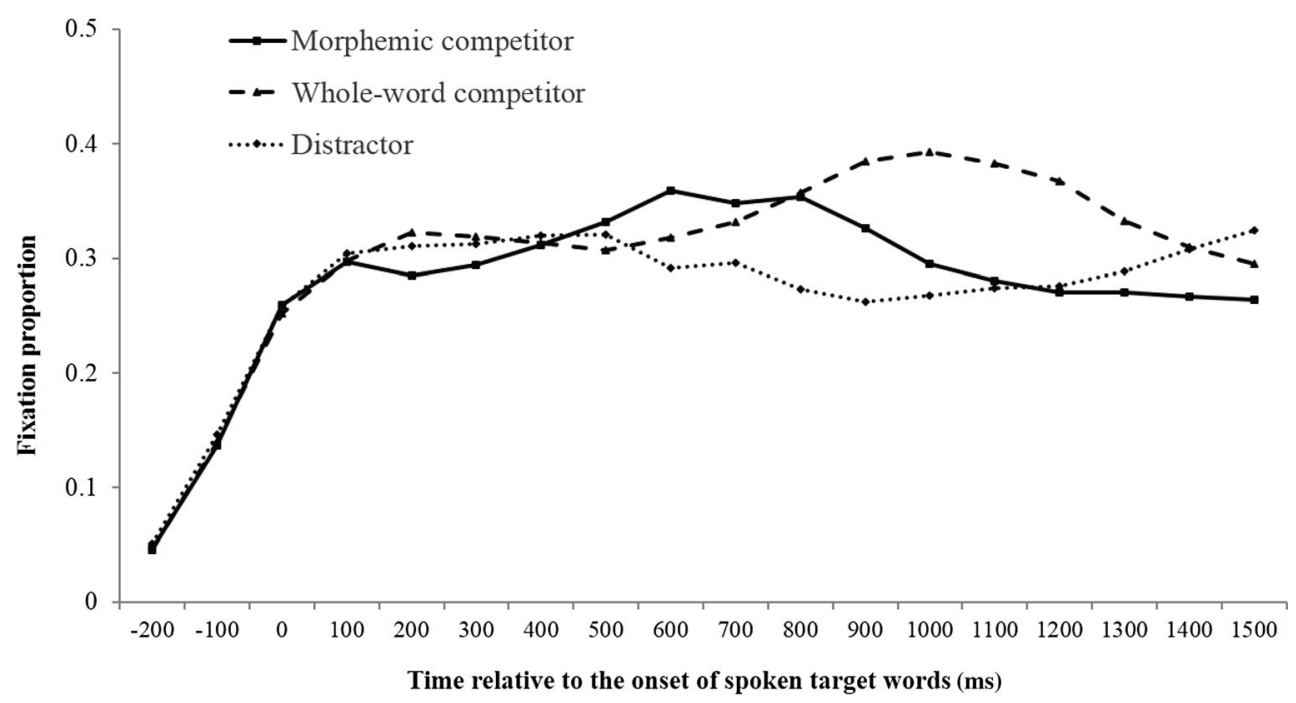

Fig. 2 Fixation proportions to morphemic competitor, whole-word competitor and unrelated distractor from $200 \mathrm{~ms}$ before onset of spoken target word

The proportion of trials with fixation to a printed word for each time window is shown in Table 1 . The results of the analysis of the logit mixed model are shown in Table 2 . These results indicated that the inclusion of a competitor did not improve model fitting in the time window of 200-300, $300-400$, and $400-500 \mathrm{~ms}$ ( $p \mathrm{~s}>.20$ ). Time-window analyses results demonstrated that the fixations on the morphemic competitor were significantly higher than those on the distractor from $600 \mathrm{~ms}$ after the onset of spoken target word. Conversely, fixations on the whole-word competitor were significantly higher than those on the distractor since $800 \mathrm{~ms}$ after the onset of spoken target word. This competitor effect emerged $200 \mathrm{~ms}$ later than the morphemic competitor effect.

\section{Discussion}

This study investigated the time course of morphological processing in spoken word recognition. Using a printed-word paradigm, we found that the morphemic competitor attracted more fixations than the distractor during spoken word recognition, demonstrating the important role that morphology plays in spoken word processing. The results of time window analyses further indicated that the divergence time point between the morphemic competitor and the distractor emerged at about $600 \mathrm{~ms}$ after the onset of the spoken target. Considering that the average duration of the first constituent was approximately $323 \mathrm{~ms}$ and that the duration of planning and executing an eye movement was about $200 \mathrm{~ms}$, the divergence point between the morphemic competitor and the distractor was almost aligned to the offset of the first constituent. This result further indicated that semantic information of the first morpheme was activated immediately when the listeners heard the first syllable. This finding provides additional evidence to support the sublexical model, which holds that morphological decomposition occurs before lexical access to the whole-word representation.

However, this morphemic competitor effect was temporary and lasted only for a short time. The finding that more fixations were made to the whole-word competitor than the

Table 1 Proportion of trials with a fixation to the visual printed words in each time window

\begin{tabular}{llll}
\hline Time window from spoken target word onset $(\mathrm{ms})$ & Morphemic competitor & Whole-word competitor & Distractor \\
\hline $200-300$ & $.33(.02)$ & $.37(.02)$ & $.36(.02)$ \\
$300-400$ & $.34(.02)$ & $.36(.01)$ & $.35(.01)$ \\
$400-500$ & $.34(.01)$ & $.34(.01)$ & $.35(.01)$ \\
$500-600$ & $.38(.01)$ & $.35(.01)$ & $.36(.01)$ \\
$600-700$ & $.40(.01)$ & $.36(.01)$ & $.33(.01)$ \\
$700-800$ & $.37(.01)$ & $.36(.01)$ & $.32(.01)$ \\
$800-900$ & $.38(.01)$ & $.38(.01)$ & $.30(.01)$ \\
$900-1000$ & $.34(.01)$ & $.41(.01)$ & $.29(.01)$ \\
\hline
\end{tabular}

Note. Standard errors are shown in parentheses 
Table 2 Logit mixed-model analyzes for competitor type

\begin{tabular}{|c|c|c|c|c|c|}
\hline Time window from spoken target word onset (ms) & Predictor & Estimate & $S E$ & $z$ values & Corrected $p$-values \\
\hline \multirow[t]{4}{*}{$600-700$} & \multicolumn{5}{|c|}{ Best fit model with competitor type: $\chi^{2}(2)=14.55, p<.001$} \\
\hline & (intercept) & -0.699 & 0.058 & -12.028 & $<.001 * * *$ \\
\hline & 0.304 & 0.081 & 3.774 & $.001 * * *$ & $<.001 * * *$ \\
\hline & 0.119 & 0.082 & 1.467 & 1.136 & 0.36 \\
\hline \multirow[t]{4}{*}{$700-800$} & \multicolumn{5}{|c|}{ Best fit model with competitor type: $\chi^{2}(2)=8.98, p=.01$} \\
\hline & (intercept) & -0.744 & 0.058 & -12.693 & $<.001 * * *$ \\
\hline & Morphemic competitor & 0.232 & 0.082 & 2.845 & $.032 *$ \\
\hline & Whole-word competitor & 0.183 & 0.082 & 2.24 & 0.192 \\
\hline \multirow[t]{4}{*}{$800-900$} & \multicolumn{5}{|c|}{ Best fit model with competitor type: $\chi^{2}(2)=28.36, p<.001$} \\
\hline & (intercept) & -0.866 & 0.059 & -14.301 & $<.001 * * *$ \\
\hline & Morphemic competitor & 0.385 & 0.082 & 4.690 & $<.001 * * *$ \\
\hline & Whole-word competitor & 0.373 & 0.082 & 4.532 & $<.001 * * *$ \\
\hline \multirow[t]{4}{*}{$900-1000$} & \multicolumn{5}{|c|}{ Best fit model with competitor type: $\chi^{2}(2)=45.29, p<.001$} \\
\hline & (intercept) & -0.918 & 0.061 & -15.136 & $<.001 * * *$ \\
\hline & Morphemic competitor & 0.276 & 0.084 & 3.292 & $.007 * *$ \\
\hline & Whole-word competitor & 0.551 & 0.082 & 6.668 & $<.001 * * *$ \\
\hline
\end{tabular}

Note. $* p<.05 . * * p<.01 . * * * p<.001$

distractor (e.g., whole-word competitor effect) suggested that the whole word was recognized quickly with more speech signals unfolding over time. Two possible mechanisms could have caused the whole word to be recognized (Packard, 1999). The first possibility is that morphemes are accessed one by one, and then the whole word is activated via a combination algorithm. The second possibility is that the first morpheme is accessed as an index to the whole-word entry. However, the current findings did not distinguish between the two possibilities because the issue is beyond the aim of our study.

Morphological processing in spoken word recognition may be language-specific. Isel et al. (2003) did not observe any semantic priming effects on the first constituent in German. Moreover, although Holle et al. (2010) provided evidence for early morphological processing in German, this finding was observed conditionally on longer German compounds. Our current finding contrasted with the findings in alphabetical languages, and one possible explanation for this difference may be language variation. Unlike in alphabetical languages, the morpheme boundary is blurry, in Chinese, morphemes are separated by spaces, making them a more salient unit. In the auditory domain, a clear syllable/morpheme boundary can also be observed because of the direct mapping between morphemes and syllables. Previous studies found that the saliency of syllables contributes to the segmentation of speech signals into individual words (Chen, Chen, \& Dell, 2002). In line with previous findings, our study shows that the saliency of Chinese syllables/morphemes facilitated the lexical decomposition, thereby enabling lexical access to the first constituent to occur immediately (Tsang \& Chen, 2014). Prosodic information could also be another possible reason for the difference. In German, the reduced duration of the first constituent often indicates the occurrence of a compound that may have caused by late morphological processing. Chinese compounds also contain a number of prosodic information, such as lexical tones and tone sandhi. Previous studies have shown that both tone category and tonal contour are activated during speech production (Nixon, Chen, \& Schiller, 2015). During spoken word recognition, listeners may use the prosodic information to select a morpheme correctly, thereby facilitating early lexical decomposition.

Previous studies also found that morphological structure can influence compound word processing. However, findings on the role of morphological structure are inconsistent (see Chung, Tong, McBride-Chang, \& Meng, 2010; Liu \& McBride-Chang, 2010). Given the unclear role of the morphological structure and the targets in this study including almost all types of morphological structures, this factor may have limited influence on our results.

Other studies have shown that concrete words are processed faster than abstract words. However, Chinese studies found that the concreteness effect can only be observed on low-frequency words (Zhang \& Zhang, 1997). Because the words used in this study were not of low frequency, this factor may exert limited influence on our results. Most morphemes used in current study were also free morphemes, which may 
have also contributed to the early lexical decomposition to some extent. However, the current findings cannot be necessarily generalized to compounds with bound morphemes.

In conclusion, our findings provide direct evidence to support the view that lexical access to spoken words is an incremental process by which listeners first access the semantic information of the first constituent immediately before they apply lexical access to the whole word during spoken word recognition. How other linguistic factors influence the morphological processing of spoken compound words remains an open question.

Author note This work was jointly funded by the National Natural Science Foundation of China (NSFC) and the German Research Foundation (DFG) in Project Crossmodal Learning, NSFC $61621136008 /$ DFC TRR-169. This research was also supported by grants from the National Natural Science Foundation of China (31571125, 31400967).

\section{References}

Barr, D. J., Levy, R., Scheepers, C., \& Tily, H. J. (2013). Random effects structure for confirmatory hypothesis testing: Keep it maximal. Journal of Memory and Language, 68(3), 255-278.

Bates, D., Maechler, M., Bolker, B., \& Walker, S. (2014). lme4: Linear mixed-effects models using Eigen and S4 (Version 1.1-7) [Computer software]. Retrieved from http://cran.r-project.org/ package $=1 m e 4$

Caramazza, A., Laudanna, A., \& Romani, C. (1988). Lexical access and inflectional morphology. Cognition, 28(3), 297-332.

Chen, J. Y., Chen, T. M., \& Dell, G. S. (2002). Word-form encoding in Mandarin Chinese as assessed by the implicit priming task. Journal of Memory and Language, 46(4), 751-781.

Chung, K. K., Tong, X., Liu, P. D., McBride-Chang, C., \& Meng, X. (2010). The processing of morphological structure information in Chinese coordinative compounds: An event-related potential study. Brain Research, 1352, 157-166.

Core Team, R. (2016). R: A language and environment for statistical computing (Version 3.3.2). Vienna: R Foundation for Statistical Computing. Retrieved from http://www.R-project.org/

Giraudo, H., \& Grainger, J. (2000). Effects of prime word frequency and cumulative root frequency in masked morphological priming. Language and Cognitive Processes, 15, 421-444.

Holle, H., Gunter, T. C., \& Koester, D. (2010). The time course of lexical access in morphologically complex words. Neuroreport, 21(5), 319-323.
Huettig, F., \& McQueen, J. M. (2007). The tug of war between phonological, semantic and shape information in language-mediated visual search. Journal of Memory and Language, 57(4), 460-482.

Hyönä, J., \& Pollatsek, A. (1998). Reading Finnish compound words: Eye fixations are affected by component morphemes. Journal of Experimental Psychology: Human Perception and Performance, 24(6), 1612-1627.

Isel, F., Gunter, T. C., \& Friederici, A. D. (2003). Prosody-assisted headdriven access to spoken German compounds. Journal of Experimental Psychology: Learning, Memory, and Cognition, 29(2), 277-288.

Jaeger, T. F. (2008). Categorical data analysis: Away from ANOVAs (transformed or not) and towards logit mixed models. Journal of Memory and Language, 59(4), 434-446.

Liu, P. D., \& McBride-Chang, C. (2010). Morphological processing of Chinese compounds from a grammatical view. Applied Psycholinguistics, 31(04), 605-617.

Nixon, J. S., Chen, Y., \& Schiller, N. O. (2015). Multi-level processing of phonetic variants in speech production and visual word processing: Evidence from Mandarin lexical tones. Language, Cognition and Neuroscience, 30(5), 491-505.

Packard, J. L. (1999). Lexical access in Chinese speech comprehension and production. Brain and Language, 68(1), 89-94.

Pollatsek, A., Hyönä, J., \& Bertram, R. (2000). The role of morphological constituents in reading Finnish compound words. Journal of Experimental Psychology: Human Perception and Performance, 26(2), 820-833.

Rastle, K., Davis, M. H., \& New, B. (2004). The broth in my brother's brothel: Morpho-orthographic segmentation in visual word recognition. Psychonomic Bulletin \& Review, 11(6), 1090-1098.

Rayner, K. (1998). Eye movements in reading and information processing: 20 years of research. Psychological Bulletin, 124(3), 372-422.

Shen, W., Qu, Q., \& Li, X. (2016). Semantic information mediates visual attention during spoken word recognition in Chinese: Evidence from the printed-word version of the visual-world paradigm. Attention, Perception, \& Psychophysics, 78(5), 1267-1284.

Taft, M., \& Forster, K. I. (1975). Lexical storage and retrieval of prefixed words. Journal of Verbal Learning and Verbal Behavior, 14(6), 638647.

Tsang, Y. K., \& Chen, H. C. (2014). Activation of morphemic meanings in processing opaque words. Psychonomic Bulletin \& Review, 21(5), $1281-1286$.

Zhang, Q., \& Zhang, B. Y. (1997). Research on concreteness effects of Chinese two-character words. Acta Psychologica Sinica, 29, 216 224. in Chinese, abstract in English.

Zhou, X., \& Marslen-Wilson, W. (1994). Words, morphemes and syllables in the Chinese mental lexicon. Language and Cognitive Processes, 9(3), 393-422.

Zhou, X., \& Marslen-Wilson, W. (2000). Lexical representation of compound words: Cross-linguistic evidence. Psychologia, 43, 47-66. 